\title{
THE LITTLE DESARGUESIAN THEOREM FOR ALGEBRAS IN MODULAR VARIETIES
}

\author{
H. PETER GUMM
}

\begin{abstract}
We show that the "Little Desarguesian Theorem" is true in the congruence-class geometry of algebras in modular varieties.
\end{abstract}

0. Introduction. In [2] R. Freese and B. Jónsson have shown that the congruence lattices of algebras in modular varieties are in fact arguesian. Since the congruence lattice of an algebra $\mathfrak{A}$ may be thought of as the (abstract) projective geometry of $\mathfrak{U}$ it is natural to ask whether there might be an affine analogue to this result.

An affine geometry, Kongruenzklassengeometrie by name, was introduced and investigated by $R$. Wille in [7]. Simply take the elements of $\mathfrak{A}$ as points and every congruence class as a subspace.

In this general setting there is no transition nor even a connection known between these two geometries. Thus it may be surprising that there is an affine analogue to the above-mentioned result of Freese and Jónsson. Namely, as we are about to prove, the "Little Desarguesian Theorem" is true in the Kongruenzklassengeometrie of algebras in modular varieties.

1. An (abstract) algebra $\mathfrak{A}$ is a set $A$ together with a family of fundamental operations. A congruence on $\mathfrak{A}$ is an equivalence relation on $A$, compatible with all fundamental operations. The congruences on $\mathfrak{A}$, ordered by set inclusion, form an algebraic lattice, denoted by $\mathfrak{S}(\mathfrak{A})$. $\mathfrak{A}$ is called congruence modular if $\mathfrak{E}(\mathfrak{A})$ is a modular lattice. As a general reference for all those notions we suggest [3]. We continue to call terms all those operations which may be constructed from the fundamental operations by successive superpositions and identifications of variables.

We start with the following trivial observation from [4]. However trivial, this lemma will be seen to be important for the sequel, so we refer to it by the name of "Shifting Lemma".

LeMma. Let $\mathfrak{A}$ be congruence modular and let $a, b, c, d$ be elements of $\mathfrak{A}$. Let $\boldsymbol{\theta}_{0}, \boldsymbol{\theta}_{1}$ and $\Psi$ be congruences on $\mathfrak{A}$ such that $(a, b) \in \theta_{0},(c, d) \in \theta_{0},(a, d) \in \theta_{1},(b, c) \in$ $\theta_{1} \wedge \Psi$. Then $(a, d) \in\left(\theta_{0} \wedge \theta_{1}\right) \vee \Psi$.

We prefer to express this lemma pictorially. We draw points for elements of $\mathfrak{U}$ and join two points, $a$ and $b$, with a line if they are congruent modulo some

Received by the editors December 21, 1979.

1980 Mathematics Subject Classification. Primary 08B10; Secondary 51A20.

Key words and phrases. Modular varieties, congruence class geometry, configurations, Desarguesian. 
congruence $\theta$, in which case we will attach the name $\theta$ to the line joining $a$ and $b$. We will draw two lines parallel just in case they both represent classes of the same congruence relation.

Thus a reformulation of the lemma would be

Shifting Lemma. Let $\mathfrak{A}$ be congruence modular, $a, b, c, d \in \mathfrak{A}, \boldsymbol{\theta}_{0}, \boldsymbol{\theta}_{\mathfrak{1}}, \Psi \in \mathfrak{(}(\mathfrak{Q})$. Then

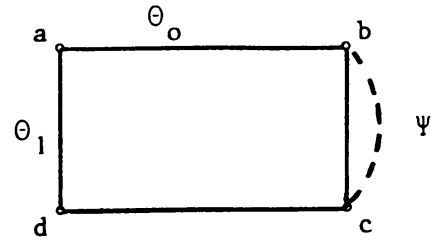

implies

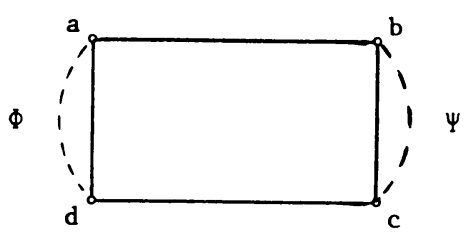

with $\Phi=\left(\theta_{0} \wedge \theta_{1}\right) \vee \Psi$.

For the rest of this note we will assume that $\mathfrak{A}$ generates a modular variety, i.e. that subalgebras of powers of $\mathfrak{A}$ are congruence modular.

2. A closure theorem. We assume that the reader has become familiar with our geometrical language, so we dare to formulate our theorem pictorially.

2.1. TheORem. Let $\mathfrak{A}$ be an algebra in a modular variety; let $x, y, z, u, x^{\prime}, y^{\prime}, z^{\prime}, u^{\prime}$ be elements of $\mathfrak{A}$ and $\theta, \Psi, \alpha_{1}, \alpha_{2}$ congruences on $\mathfrak{A}$ with $\theta \wedge \alpha_{i}<\Psi$ for $i=1,2$. Then
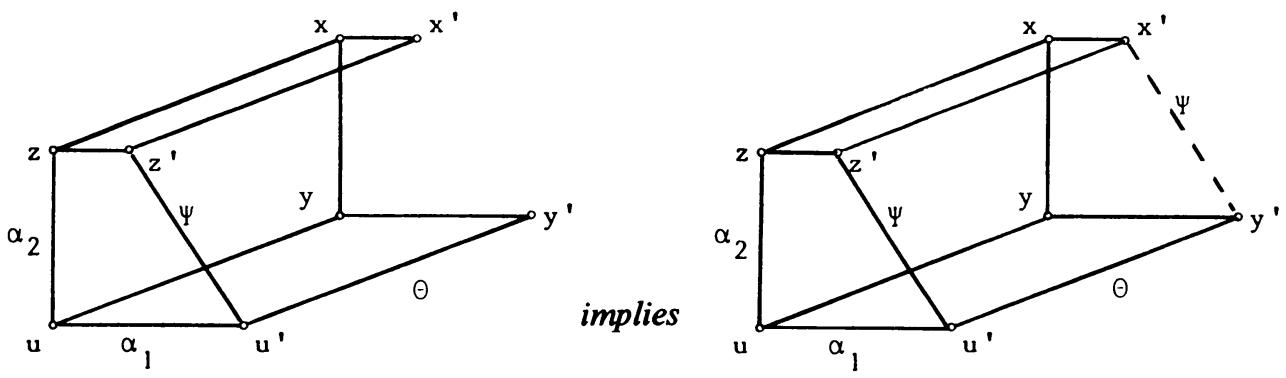

Proof. According to a theorem of A. Day [1] there exists a natural number $\boldsymbol{n}$, and four-ary terms $\underline{m}_{0}, \underline{m}_{1}, \ldots, \underline{m}_{n}$ such that the following equations are satisfied for arbitrary $a, b, c, d \in \mathfrak{A}$.

$$
\begin{aligned}
& \underline{m}_{0}(a, b, c, d)=a, \quad \underline{m}_{n}(a, b, c, d)=b . \\
& \underline{m}_{i}(a, a, c, c)=a \text { for all } i<n . \\
& \underline{m}_{i}(a, b, a, b)=\underline{m}_{i+1}(a, b, a, b) \text { for } i \text { even. } \\
& \underline{m}_{i}(a, b, c, c)=\underline{m}_{i+1}(a, b, c, c) \text { for } i \text { odd. }
\end{aligned}
$$

For all $i$ we now define points $m_{i}$ and $\bar{m}_{i}$ by

$$
m_{i}:=\underline{m}_{i}\left(x^{\prime}, y^{\prime}, z^{\prime}, u^{\prime}\right), \quad \bar{m}_{i}:=\underline{m}_{i}(x, y, z, u) .
$$

Equations (I) and (IV) immediately yield

$$
m_{0}=x^{\prime}, \quad m_{n}=y^{\prime}
$$


and

$$
m_{i} \Psi m_{i+1} \text { for } i \text { odd. }
$$

The relations

$$
m_{i} \theta m_{i+1} \text { for } i \text { even }
$$

and

$$
\bar{m}_{i} \theta \bar{m}_{i+1} \text { for } i \text { even }
$$

follow from (III). Also important are

$$
\bar{m}_{i} \alpha_{2} \bar{m}_{i+1} \text { for all } i \text { (because of (II)) }
$$

and

$$
m_{i} \alpha_{1} \bar{m}_{i} \text { for all } i
$$

as a consequence of the definition of $m_{i}$ and $\bar{m}_{i}$.

As a result of (3), (3), (4) and (5) we have for every even $i$ the familiar configuration:

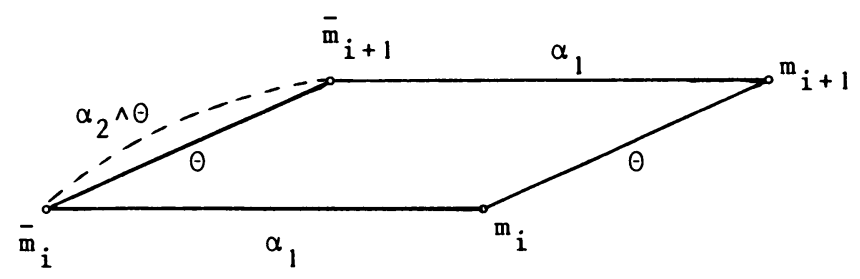

Applying the Shifting Lemma we obtain

$$
\left(m_{i}, m_{i+1}\right) \in\left(\theta \wedge \alpha_{1}\right) \vee\left(\theta \wedge \alpha_{2}\right)
$$

and with our assumptions

$$
m_{i} \Psi m_{i+1} \text { for } i \text { even. }
$$

The result, $x^{\prime}, \Psi y^{\prime}$, follows now using transitivity and (1), (2) and (7).

Two corollaries of the theorem just proven are of special importance. Firstly, identifying $z$ with $z^{\prime}$ and $x$ with $x^{\prime}$ we obtain

2.2. The Little Desarguesian Theorem. Let $\mathfrak{A}$ be an algebra in a modular variety; let $x, y, z, x^{\prime}, y^{\prime}, z^{\prime}$ be elements of $\mathfrak{A}$ and $\theta, \Psi, \alpha_{1}, \alpha_{2}$ congruences on $\mathfrak{A}$ with $\theta \wedge \alpha_{i} \leqslant \Psi$ for $i=1,2$. Then

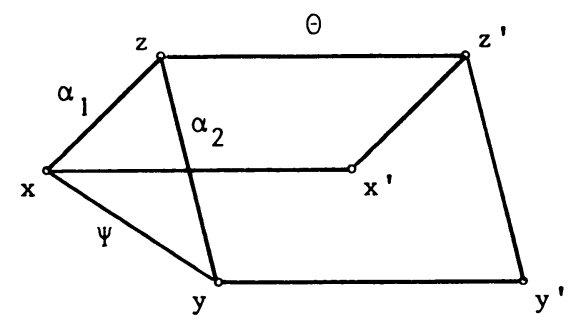

implies

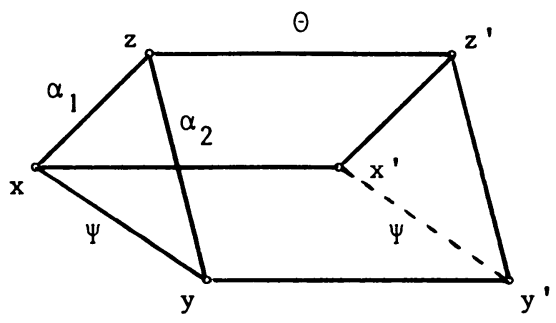


Secondly, for $\Psi=\alpha_{2}$, we obtain the

2.3. Cube Lemma. Let $\mathfrak{A}$ be an algebra in a modular variety and $\theta_{0}, \theta_{1}, \Psi$ be congruences on $\mathfrak{A}$ with $\theta_{0} \wedge \theta_{1} \leqslant \Psi$. Let $x, y, z, u, x^{\prime}, y^{\prime}, z^{\prime}, u^{\prime}$ be elements of $\mathfrak{A}$. Then
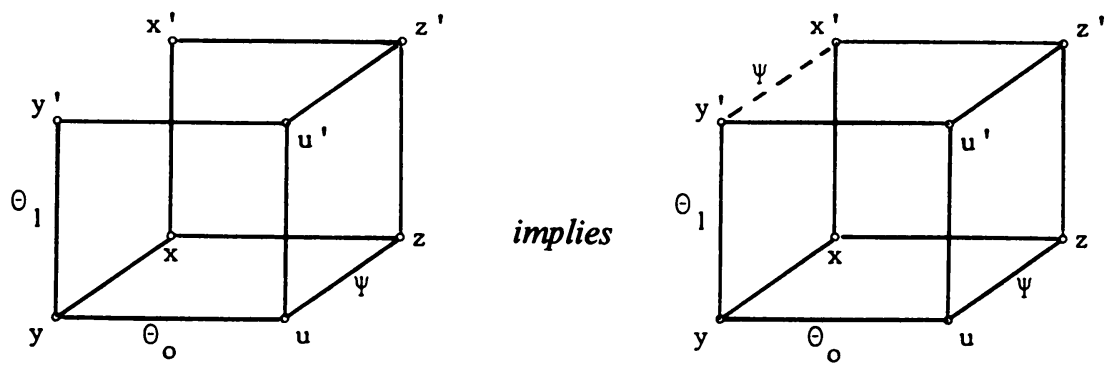

REMarK. The Cube Lemma is of particular importance in [5], where it is needed to show commutativity of the commutator operation $[\alpha, \beta]$. Note also that the Cube Lemma is a considerable strengthening of the Reidemeister Theorem which was proven for permutable varieties in [6] and for modular varieties in [4].

We may employ the same method as before to get a different closure theorem.

2.4. THEOREM. With the assumptions of Theorem 2.1 we have
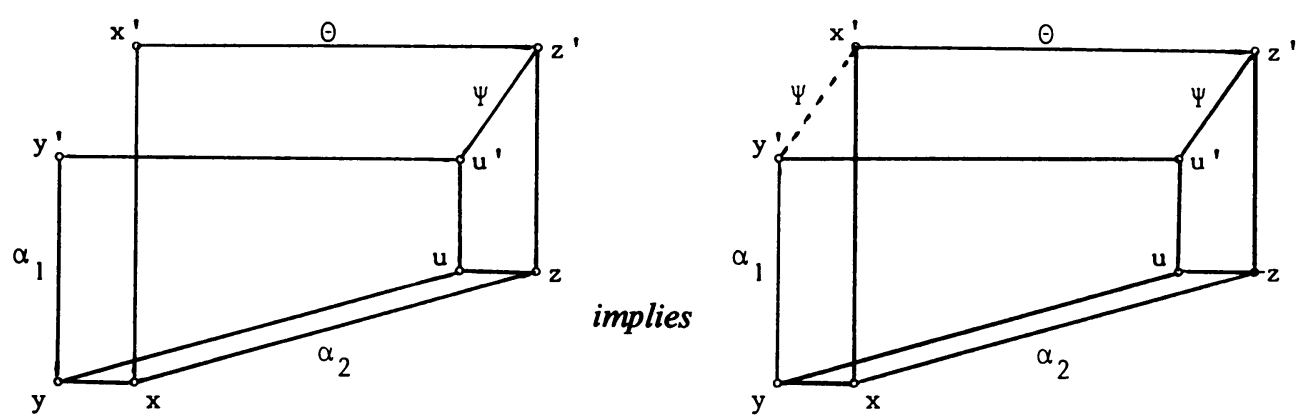

Proof. The proof is the same as the proof of Theorem 2.1 except that we have to replace the relations $(\overline{3})$ and (4) by the relations

$$
\bar{m}_{i} \alpha_{2} \bar{m}_{i+1} \text { for } i \text { even }
$$

and

$$
\bar{m}_{i} \theta \bar{m}_{i+1} \text { for all } i \text {. }
$$

Note that the last theorem yields us the closure of the Desargues configuration (in the terminology of [4]) if we identify $u$ with $u^{\prime}$ and $\Psi$ with $\alpha_{2}$. 


\section{REFERENCES}

1. A. Day, A characterization of modularity for congruence lattices of algebras, Canad. Math. Bull. 12 (1969), 167-173.

2. R. Freese and B. Jónsson, Congruence modularity implies the Arguesian identity, Algebra Universalis 6 (1976), 225-228.

3. G. Grätzer, Universal algebra, Van Nostrand, Princeton, N. J., 1968.

4. H. P. Gumm, Über die Läsungsmengen von Gleichungssystemen über allgemeinen Algebren, Math. Z. 162 (1978), 51-62.

5. , An easy way to the commutator in modular varieties, Arch. Math. (to appear).

6. Algebras in permutable varieties: Geometrical properties of affine algebras, Algebra Universalis 9 (1979), 8-34.

7. R. Wille, Kongruenzklassengeometrien, Lecture Notes in Math., vol. 113, Springer-Verlag, Berlin and New York, 1970.

Techische Hochschule, Darmstadt, Federal Republic of Germany 